\section{ISSN 1991- 8690}

Website: http://jsci.utq.edu.iq

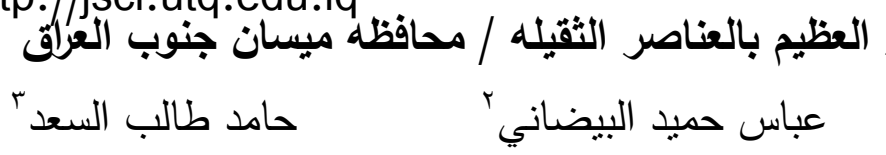

$$
\text { r قسم كيمياء البيئة البحرية ـ مركز علوم البحار - جامعة البصرة / علبة العلوم العراق }
$$

الترقيم الدولي • 1991 ـ 19

Email: utjsci@utq.edu.iq

تقيم ثلوث رواسب هور

اقبال عوفي الطائي'

الخلاصه

قيست تراكيز بعض العناصرالثقبله ( الرصاص ، الحديد، النحاس، النيكل و الكروم ) لرواسب لبابية في محطنين من هور العظيم ضمن هورالحويزة

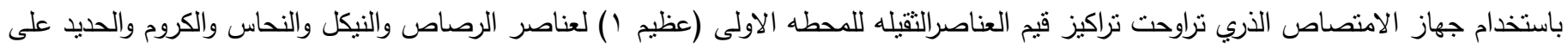

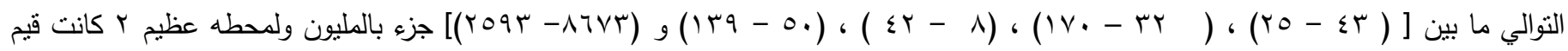

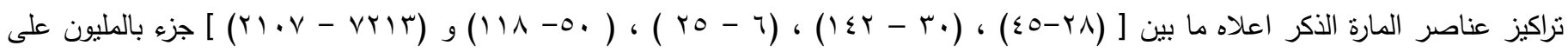

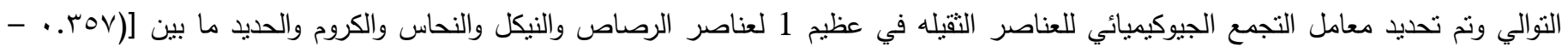

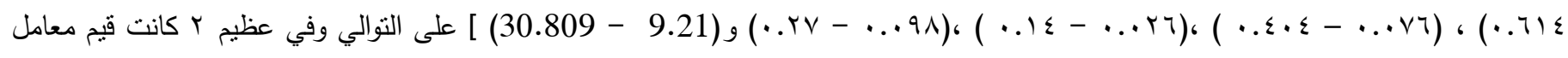

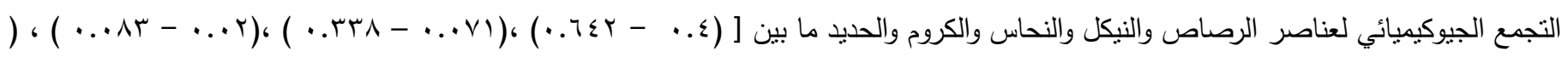

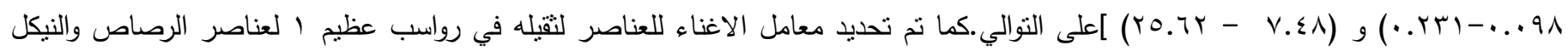

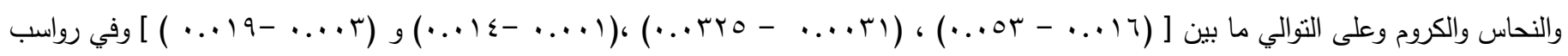

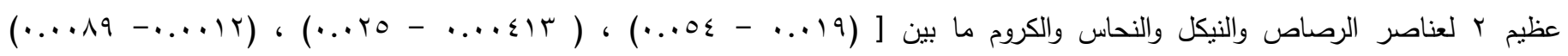

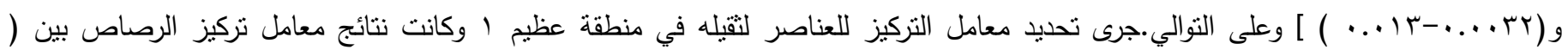

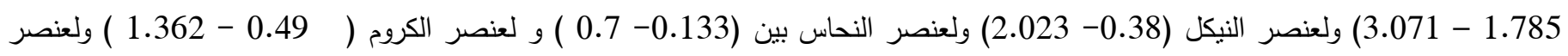

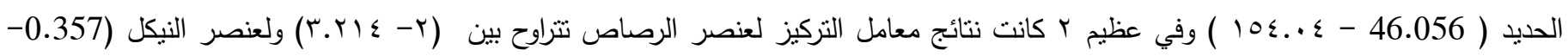

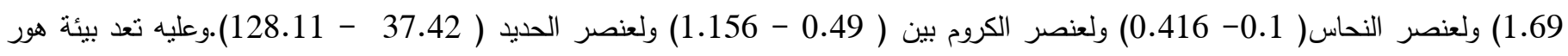

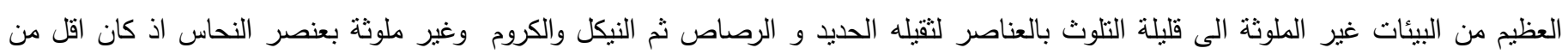

\title{
Evalution of Pollution in the Hor AL -Azim Sediments by Heavy metals/ Missan Government / South of Iraq
}

\section{Abstract}

The current study was included measurementof concentrations the coefficients of heavy elements in sediments of casement in Hor AL-Azim reality within Hwaiza Marshes in two area Azim1 andAzim 2. measured concentrations of some heavy metals in sediments core of the study area lead, nickel, copper, Chrome and iron, appeared concentrations of values in Azim 1 (43 - 25) ppm, (32 - 170) ppm,copper (8 - 42) ppm and (50 - 139) ppm and (2593 - 8675) ppm respectively. Also been identified coefficient assembly geochemical heavy metals, which was in Azim 1 for lead ,nickel,copper,Chrome, iron between $(0.357$ - 0.585), (0.076 - 0.383), (0.026 - 0.14), (0.098 - 0.272) and (9.211 30.809) respectively, either in Azim 2 for the same metals $(0.4-0.642),(0.071-0.338),(0.02-0.083),(0.098-0.231)$ and (7.48 - 25.62) respectively. Also determined the coefficient enrichment of heavy metals in the sediments of Azim 1 for lead ,nickel,copper,Chromebetween $(0.016$ - 0.053), $(0.0031-0.0325),(0.001-0.014)$ and $(0.003-0.019)$ respectively and Azim 2 for the same metals between $(0.019$ - 0.054), (0.00413-0.025), (0.0012 -0.0089) and (0.00320.013) respectively. Identified Concentration Factor of the heavy metals in the Azim 1 for lead 
,nickel,copper,Chrome, iron between(1.785-3.071), (0.38 -2.023),(0.7-0.133), (0.49-1.362) and (46.056 - 154.04) and Azim 2 for the same metals between $(2-3.214),(0.357-1.69),(0.1-0.416),(0.49-1.156)$ and $(37.424-128.11)$ respectively.

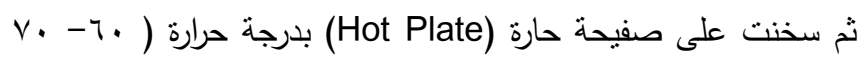
المقدمة م· ) ثم بردت قبل ان تصل الى الجفاف يبرد الراسب واضيف إليه

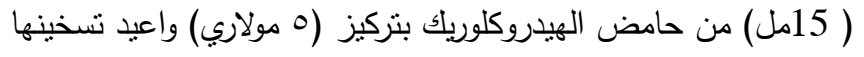

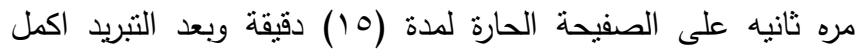
الحجم بإضافة حامض الهيدروكلوريك بتركيز (ه مولاري) إلى ( 25

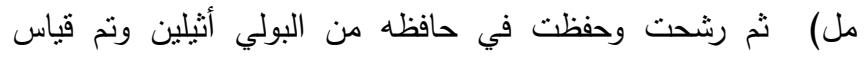

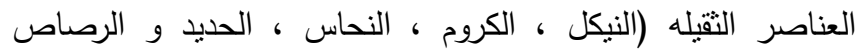

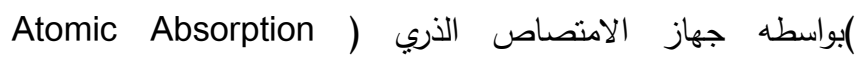
- ( Spectrophotometer

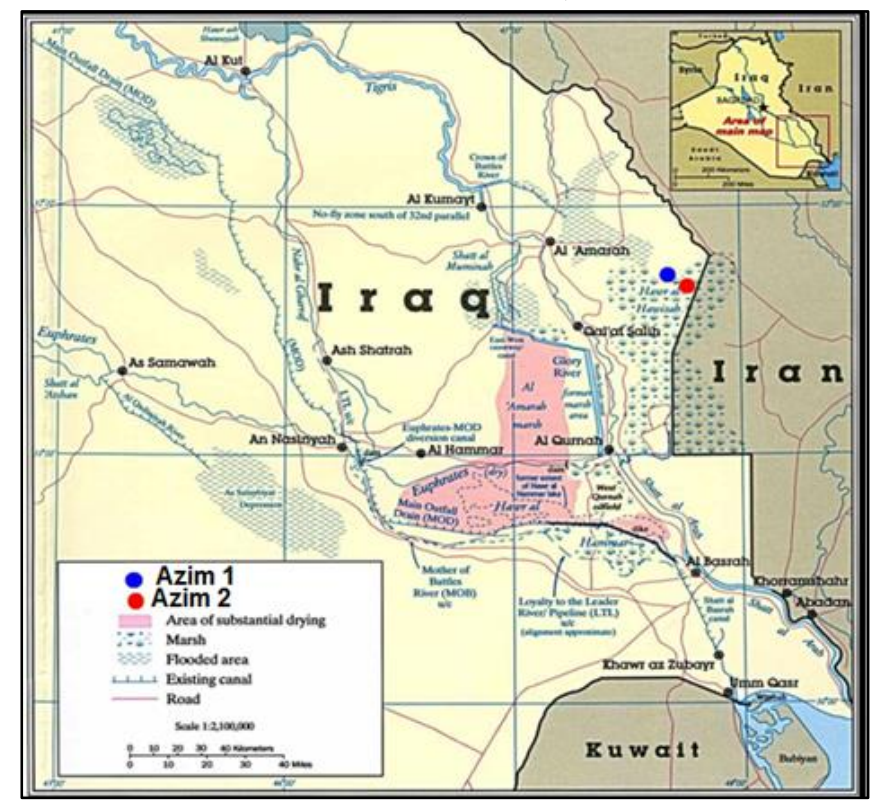

شكل( (1) خارطة لموقع منطقة الدراسة

\section{النتائجج}

قيست نزاكيز العناصر الثقيله لكل من عنصر الرصاص والنيكل والنحاس والكروم والحديد في عينات الدراسة اذ سجلت اعلى التركيز في

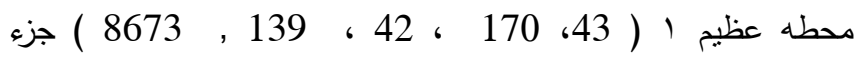

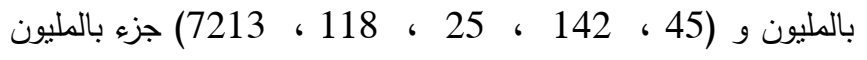

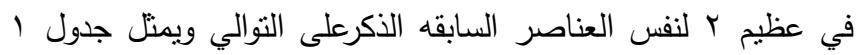
تراكيز العناصر الثقيله للعينات اللبابيه في عظيم ا ويمثل جدول بلني تزاكيز العناصر التقيله للعينات اللبابيه في عظيم r .
تعد العناصر التقيلة ذات اهمية بالغة في مجال التلوث البيئي لما لها من ناثيرات عديدة على البيئة سواء كانت بيئة رسوبية او بيئه مائية

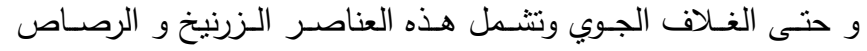

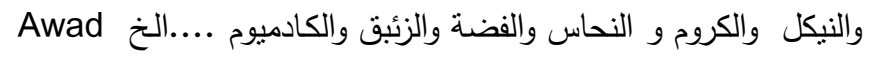

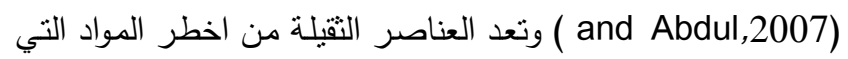

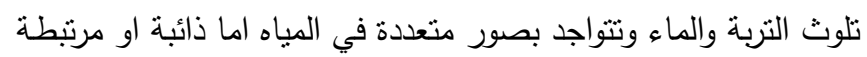
مع المواد العضوية بشكل معقدات او دقائق وتتنقل من مصدر تلوثنها ونياه

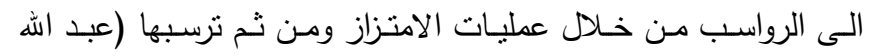

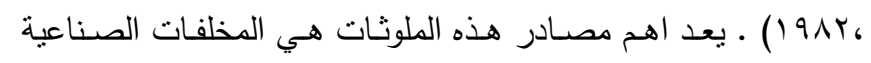

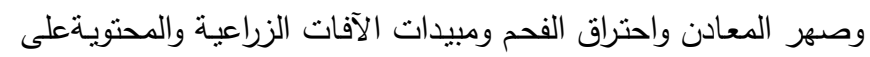

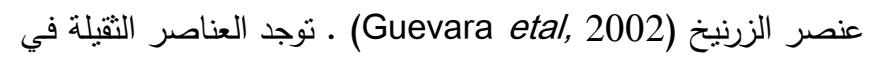
جميع الرواسب والمياه كما تدخل بعض هذه العناصر عدا الكادميوم

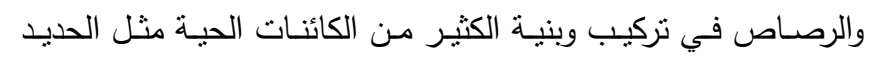

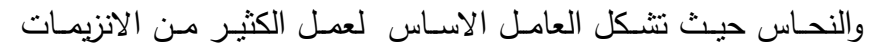

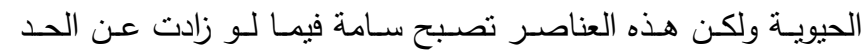

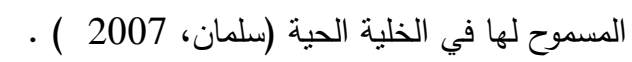
المواد وطرائق العمل اخذت النماذج في السابع من ديسمبر لمنطقه هور العظيم الواقع شمال شرق هور الحويزة وجرى اختيار محطنتين باستخدام جهاز الـ E ) هي عظيم 1 عند الموقع ("N $31^{0} 42$ (GPS)

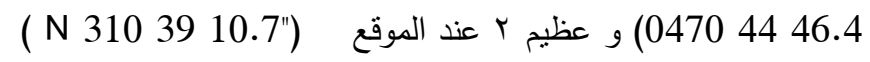

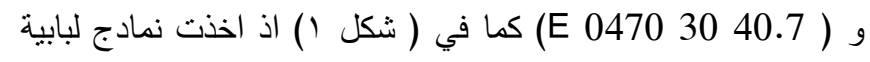

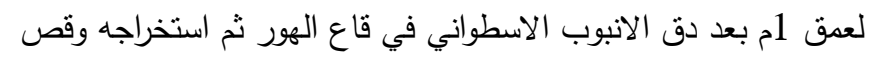

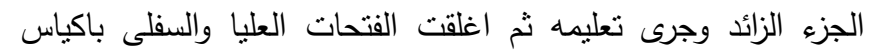

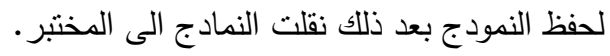
العمل المختبري

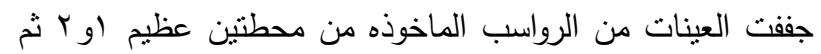

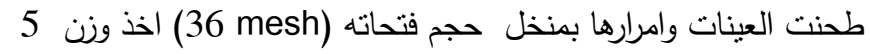
غرامات من كل عينه و وضعت في بيكر زجاجي سعة ( 100مل)

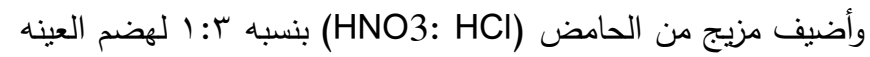




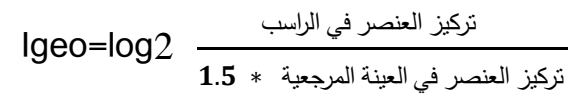

ويمثل جدول (بو ع) قيم معامل التجمع الجيوكيميائي لمحطه عظيم

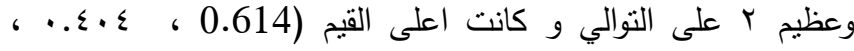
ع 0.338 ، 0.083 ، , آr.. 25.623) لمحطه عظيم r لعنصر الرصاص والنيكل والنحاس والكروم والحديد على التوالي

$$
\text { جدول (r) قيم معامل التجمع الجيوكيميائي في عظيم ا }
$$

\begin{tabular}{|c|c|c|c|c|c||}
\hline (2- أعنق & Igeo pb & Igeo Ni & Igeo Cu & Igeo Cr & Igeo Fe \\
\hline $0-5$ & 0.557 & 0.142 & 0.05 & 0.098 & 30.809 \\
\hline $5-10$ & 0.571 & 0.195 & 0.06 & 0.127 & 29.811 \\
\hline $10-15$ & 0.471 & 0.295 & 0.083 & 0.188 & 27.648 \\
\hline $15-20$ & 0.471 & 0.3 & 0.073 & 0.192 & 26.579 \\
\hline $20-25$ & 0.557 & 0.1 & 0.03 & 0.098 & 27.136 \\
\hline $25-30$ & 0.585 & 0.088 & 0.03 & 0.098 & 23.193 \\
\hline $30-35$ & 0.571 & 0.076 & 0.026 & 0.098 & 23.825 \\
\hline $35-40$ & 0.585 & 0.085 & 0.026 & 0.098 & 20.422 \\
\hline $40-45$ & 0.614 & 0.107 & 0.033 & 0.098 & 14.923 \\
\hline $45-50$ & 0.514 & 0.309 & 0.11 & 0.215 & 16.316 \\
\hline $50-55$ & 0.357 & 0.404 & 0.123 & 0.272 & 22.163 \\
\hline $55-60$ & 0.357 & 0.383 & 0.12 & 0.254 & 21.676 \\
\hline $60-65$ & 0.357 & 0.209 & 0.083 & 0.119 & 13.520 \\
\hline $65-70$ & 0.357 & 0.183 & 0.076 & 0.098 & 19.040 \\
\hline $70-75$ & 0.357 & 0.183 & 0.083 & 0.098 & 17.527 \\
\hline $75-80$ & 0.557 & 0.126 & 0.04 & 0.098 & 17.232 \\
\hline $80-85$ & 0.528 & 0.104 & 0.033 & 0.098 & 13.538 \\
\hline $85-90$ & 0.557 & 0.102 & 0.033 & 0.098 & 10.493 \\
\hline $90-95$ & 0.357 & 0.197 & 0.086 & 0.121 & 11.818 \\
\hline $95-100$ & 0.357 & 0.302 & 0.14 & 0.188 & 9.6269 \\
\hline $100-105$ & 0.357 & 0.3 & 0.136 & 0.158 & 9.2113 \\
\hline AV. & 0.476 & 0.199 & 0.07 & 0.138 & 19.358 \\
\hline & & & & & \\
\hline
\end{tabular}

جدول (1) تراكيزالعناصر الثقيلة للعينات اللبابية في عظيم ا جزء

\begin{tabular}{|c|c|c|c|c|c|}
\hline العمتى (س-) & $\mathbf{P b}$ & $\mathbf{N i}$ & $\mathrm{Cu}$ & $\mathrm{Cr}$ & $\mathbf{F e}$ \\
\hline $0-5$ & 39 & 60 & 15 & 50 & 8673 \\
\hline $5-10$ & 40 & 82 & 18 & 65 & 8392 \\
\hline $10-15$ & 33 & 124 & 25 & 96 & 7783 \\
\hline $15-20$ & 33 & 126 & 22 & 98 & 7482 \\
\hline $20-25$ & 39 & 42 & 9 & 50 & 7639 \\
\hline $25-30$ & 41 & 37 & 9 & 50 & 6529 \\
\hline $30-35$ & 40 & 32 & 8 & 50 & 6707 \\
\hline $35-40$ & 41 & 36 & 8 & 50 & 5749 \\
\hline $40-45$ & 43 & 45 & 10 & 50 & 4201 \\
\hline 45-50 & 36 & 130 & 33 & 110 & 4593 \\
\hline $50-55$ & 25 & 170 & 37 & 139 & 6239 \\
\hline $55-60$ & 25 & 161 & 36 & 130 & 6102 \\
\hline $60-65$ & 25 & 88 & 25 & 61 & 3806 \\
\hline $65-70$ & 25 & 77 & 23 & 50 & 5360 \\
\hline $70-75$ & 25 & 77 & 25 & 50 & 4934 \\
\hline $75-80$ & 39 & 53 & 12 & 50 & 4851 \\
\hline $80-85$ & 37 & 44 & 10 & 50 & 3811 \\
\hline $85-90$ & 39 & 43 & 10 & 50 & 2954 \\
\hline $90-95$ & 25 & 83 & 26 & 62 & $\mathbf{3 3 2 7}$ \\
\hline $95-100$ & 25 & 127 & 42 & 96 & 2710 \\
\hline $100-105$ & 25 & 126 & 41 & $\mathbf{8 1}$ & 2593 \\
\hline
\end{tabular}
بالمليون

جدول (Y) تراكيزالعناصر الثقيله للعينات اللبابية في عظيم Y جزء

\begin{tabular}{|c|c|c|c|c|c|}
\hline العتق ( - (- & $\mathrm{Pb}$ & Ni & $\mathrm{Cu}$ & $\mathrm{Cr}$ & $\mathbf{F e}$ \\
\hline $0-5$ & 45 & 67 & 16 & 50 & 7213 \\
\hline $5-10$ & 41 & 85 & 17 & 72 & 7049 \\
\hline $10-15$ & 3.8 & 96 & 19 & 83 & 6904 \\
\hline $15-20$ & 3.4 & 102 & 21 & 82 & 6743 \\
\hline $20-25$ & 33 & 142 & 25 & 118 & 6683 \\
\hline $25-30$ & 31 & 142 & 25 & 112 & 5908 \\
\hline $30-35$ & 32 & 142 & 25 & 115 & 5862 \\
\hline $35-40$ & 33 & 140 & 25 & 114 & 5830 \\
\hline $40-45$ & 38 & 36 & 8 & 50 & 5829 \\
\hline $45-50$ & 43 & 3.4 & 8 & 50 & 4901 \\
\hline $50-55$ & 41 & 33 & 8 & 50 & 4722 \\
\hline $55-60$ & 37 & 30 & 6 & 50 & 4103 \\
\hline $60-65$ & 37 & 42 & 9 & 50 & 3225 \\
\hline $65-70$ & 38 & 56 & 13 & 50 & 3045 \\
\hline $70-75$ & 3,4 & 54 & 13 & 50 & 2967 \\
\hline $75-80$ & 32 & 59 & 15 & 50 & 2356 \\
\hline $80-85$ & 28 & 80 & 20 & 62 & 2107 \\
\hline
\end{tabular}
بالمليون

كما قيست معاملات التجمع الجيوكيميائي وهو دالة لمدى ثلوث الرواسب بالعناصر الثقيلة وقد استخدم من قبل Muller (1979) أذ اعتمد على تراكيز العناصر في القترة الارضية كتركيز مرجعي للعناصر وذلك لصعوبة الحصول على عينة مرجعية نتيجه لزيادة الانشطه البشرية و حسب معامل التجمع الجيوكيميائي من القانون • التالي 
جدول (0) قيم معامل الاغناء في عظيم

\begin{tabular}{|c|c|c|c|c|}
\hline "لمتق (-2) & EF Cr & EF Cu & EF $\mathrm{Ni}$ & EF Pb \\
\hline $0-5$ & 0.0031 & 0.0016 & 0.0046 & 0.018 \\
\hline $5-10$ & 0.0042 & 0.0020 & 0.0065 & 0.0191 \\
\hline $10-15$ & 0.0068 & 0.0030 & 0.0106 & 0.017 \\
\hline $15-20$ & 0.0072 & 0.0027 & 0.0112 & 0.0177 \\
\hline $20-25$ & 0.0036 & 0.0011 & 0.0036 & 0.0205 \\
\hline $25-30$ & 0.0042 & 0.0012 & 0.0037 & 0.0252 \\
\hline $30-35$ & 0.0041 & 0.0011 & 0.0031 & 0.0239 \\
\hline $35-40$ & 0.0048 & 0.0013 & 0.0041 & 0.0286 \\
\hline $40-45$ & 0.0065 & 0.0022 & 0.0071 & 0.0411 \\
\hline $45-50$ & 0.0132 & 0.0067 & 0.0189 & 0.0315 \\
\hline $50-55$ & 0.0122 & 0.0055 & 0.0182 & 0.0161 \\
\hline $55-60$ & 0.0117 & 0.0055 & 0.0176 & 0.0164 \\
\hline $60-65$ & 0.0088 & 0.0061 & 0.0154 & 0.0264 \\
\hline $65-70$ & 0.0051 & 0.0040 & 0.0096 & 0.0187 \\
\hline $70-75$ & 0.0055 & 0.0047 & 0.010 & 0.0203 \\
\hline $75-80$ & 0.0056 & 0.0023 & 0.0073 & 0.0323 \\
\hline $80-85$ & 0.0072 & 0.0024 & 0.0077 & 0.0390 \\
\hline $85-90$ & 0.0093 & 0.0031 & 0.0097 & 0.0530 \\
\hline $90-95$ & 0.0102 & 0.0073 & 0.0167 & 0.0302 \\
\hline $95-100$ & 0.0195 & 0.0145 & 0.031 & 0.0370 \\
\hline $100-105$ & 0.0172 & 0.0148 & 0.0325 & 0.0387 \\
\hline
\end{tabular}

جدول (T) قيم معامل الاغناء في عظيم r

\begin{tabular}{||c|c|c|c|c|}
\hline$(-\lambda i n$ & EF (Pb) & EF (Ni) & FF (Cr) & FF (Cu) \\
\hline $0-5$ & 0.0250 & 0.0062 & 0.0032 & 0.0020 \\
\hline $5-10$ & 0.023 & 0.008 & 0.0047 & 0.0022 \\
\hline $10-15$ & 0.0221 & 0.0093 & 0.0056 & 0.0025 \\
\hline $15-20$ & 0.0202 & 0.0101 & 0.0057 & 0.0029 \\
\hline $20-25$ & 0.0198 & 0.0142 & 0.0082 & 0.0035 \\
\hline $25-30$ & 0.0211 & 0.0161 & 0.0088 & 0.0039 \\
\hline $30-35$ & 0.0219 & 0.0162 & 0.0092 & 0.0040 \\
\hline $35-40$ & 0.0227 & 0.0160 & 0.0091 & 0.0040 \\
\hline $40-45$ & 0.0262 & 0.0041 & 0.0040 & 0.0012 \\
\hline $45-50$ & 0.0352 & 0.0046 & 0.0047 & 0.0015 \\
\hline $50-55$ & 0.0349 & 0.0046 & 0.0049 & 0.0015 \\
\hline $55-60$ & 0.0362 & 0.0049 & 0.0057 & 0.0013 \\
\hline $60-65$ & 0.0461 & 0.0087 & 0.0072 & 0.0026 \\
\hline $65-70$ & 0.0501 & 0.0123 & 0.0077 & 0.0040 \\
\hline $70-75$ & 0.0460 & 0.0121 & 0.0079 & 0.0041 \\
\hline $75-80$ & 0.054 & 0.0167 & 0.0099 & 0.0059 \\
\hline $80-85$ & 0.053 & 0.0254 & 0.0138 & 0.00890 \\
\hline
\end{tabular}

$$
\text { جدول (§) قيم معامل التجمع الجيوكيميائي في عظيم r }
$$

\begin{tabular}{||c|c|c|c|c|c|}
\hline (2-) & Igeo $\mathrm{pb}$ & Igeo $\mathrm{Ni}$ & Ige0 $\mathrm{Cu}$ & Igeo $\mathrm{Cr}$ & Igeo .Fe \\
\hline $0-5$ & 0.642 & 0.159 & 0.053 & 0.098 & 25.623 \\
\hline $5-10$ & 0.585 & 0.202 & 0.056 & 0.141 & 25.04 \\
\hline $10-15$ & 0.542 & 0.228 & 0.063 & 0.162 & 24.525 \\
\hline $15-20$ & 0.485 & 0.242 & 0.07 & 0.160 & 23.953 \\
\hline $20-25$ & 0.471 & 0.338 & 0.083 & 0.231 & 23.740 \\
\hline $25-30$ & 0.442 & 0.338 & 0.083 & 0.219 & 20.987 \\
\hline $30-35$ & 0.457 & 0.338 & 0.083 & 0.225 & 20.824 \\
\hline $35-40$ & 0.471 & 0.333 & 0.083 & 0.223 & 20.710 \\
\hline $40-45$ & 0.542 & 0.085 & 0.026 & 0.098 & 20.706 \\
\hline $45-50$ & 0.614 & 0.08 & 0.026 & 0.098 & 17.410 \\
\hline $50-55$ & 0.585 & 0.078 & 0.026 & 0.098 & 16.774 \\
\hline $55-60$ & 0.528 & 0.071 & 0.02 & 0.098 & 14.575 \\
\hline $60-65$ & 0.528 & 0.1 & 0.03 & 0.098 & 11.456 \\
\hline $65-70$ & 0.542 & 0.133 & 0.043 & 0.098 & 10.817 \\
\hline $70-75$ & 0.485 & 0.128 & 0.043 & 0.098 & 10.539 \\
\hline $75-80$ & 0.457 & 0.140 & 0.05 & 0.098 & 8.3694 \\
\hline $80-85$ & 0.4 & 0.190 & 0.066 & 0.121 & 7.4849 \\
\hline AV. & 0.516 & 0.187 & 0.053 & 0.139 & 17.855 \\
\hline
\end{tabular}

لتقيم (EF) ( enrichment factor) لوحسب معامل الاغناء معدل التلوث في البيئة وقد تم استخدام تركيز الحديد كونه الآكثر انتشارا في القشرة الارضية ، وكما في المعادلة التالية :

(CM/CFe)Earth,s crust / EF=(CM/CFe)sample اذ يمثل البسط نركيز العناصر في العينات الرسوبية بينما بمثل المقام تركيز العناصر في القشرة الارضية يوضح جدول (0) قيم معامل

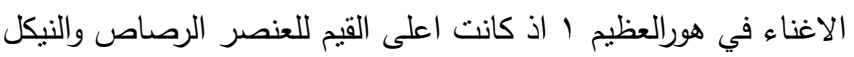
والنحاس والكروم على التوالي ( 0.0530 ( 0.0325 ، 0.032 ، 0.0148 ، 0.0195 ) · جدول (؟) يمثل قيم معامل الاغناء للعناصر الثقيلة في

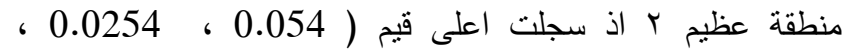
عالق

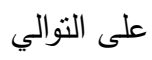


جدول (^) قيم معامل النلوث في عظيم r

\begin{tabular}{|c|c|c|c|c|c|}
\hline العقى (س) & الترصاص CF & الكروم CF & التحاسنCF & التيكل CF & CF.الحطيد \\
\hline $0-5$ & 3.214 & 0.490 & 0.266 & 0.797 & 128.11 \\
\hline 5-10 & 2.928 & 0.705 & 0.283 & 1.011 & 125.2 \\
\hline $10-15$ & 2.714 & 0.813 & 0.316 & 1.142 & 122.62 \\
\hline $15-20$ & 2.428 & 0.803 & 0.35 & 1.214 & 119.76 \\
\hline $20-25$ & 2.357 & 1.156 & 0.416 & 1.690 & 118.70 \\
\hline $25-30$ & 2.214 & 1.098 & 0.416 & 1.690 & 104.93 \\
\hline 30-35 & 2.285 & 1.127 & 0.416 & 1.690 & 104.12 \\
\hline $35-40$ & 2.357 & 1.117 & 0.416 & 1.666 & 103.55 \\
\hline $40-45$ & 2.714 & 0.490 & 0.133 & 0.428 & 103.53 \\
\hline $45-50$ & 3.071 & 0.490 & 0.133 & 0.404 & 87.05 \\
\hline 50-55 & 2.928 & 0.490 & 0.133 & 0.392 & 83.87 \\
\hline $55-60$ & 2.642 & 0.490 & 0.1 & 0.357 & 72.87 \\
\hline $60-65$ & 2.642 & 0.490 & 0.15 & 0.5 & 57.28 \\
\hline $65-70$ & 2.714 & 0.490 & 0.216 & 0.666 & 54.08 \\
\hline $70-75$ & 2.428 & 0.490 & 0.216 & 0.642 & 52.69 \\
\hline $75-80$ & 2.285 & 0.490 & 0.25 & 0.702 & 41.84 \\
\hline $80-85$ & 2 & 0.607 & 0.333 & 0.952 & 37.42 \\
\hline
\end{tabular}

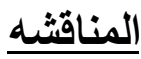

تتضاعف تراكيزالعناصر الثقيلة في المدن الصناعية مماعليه في

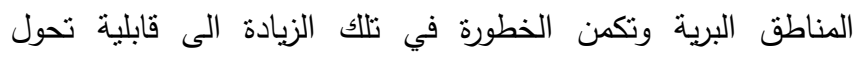
العتاصر النقيلة الى مركبات سمية بمرور الزمن والتي يعد وجودها

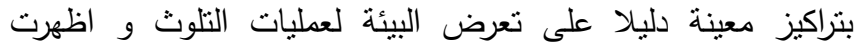

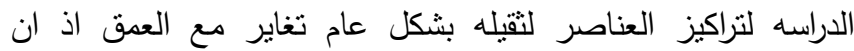

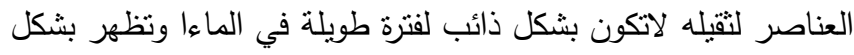

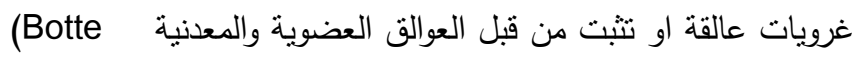
تكون الكتيونات الذائبة من العناصر التقبله سهلة

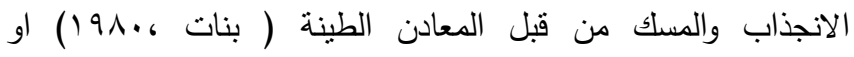

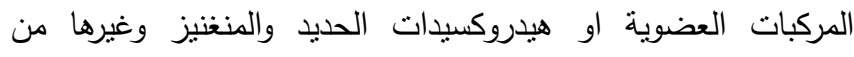

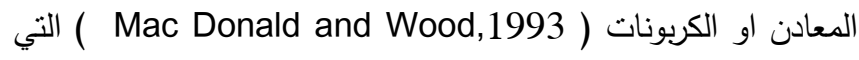
تتجمع على الرواسب السطحية او تمتص من قبل النباتات المائية ان هذا التغاير ناتج من الاختلاف في نسب كل من الطين والغرين والرمل فضلا عن الاختلاف في المحتوى العضوي لكل طبقة اذ يزداد امتزاز

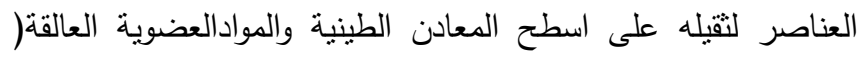
بنات ، •9191)، وقد جاء تزايد التزاكيز للعناصر الثقيلة مرافقة لمناطق زيادة نسبة الاطيان والغرين في العمود الرسوبي لمنطقة هور العظيم

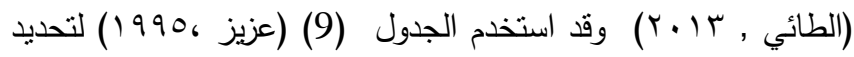

كمانم احتساب عامل التلوث (CF) وهو احد اهم المعايير المعبرة عن

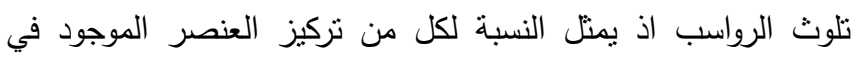

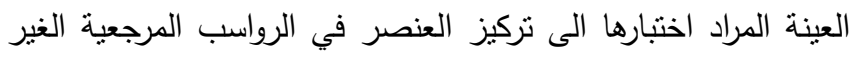

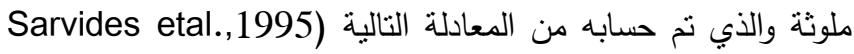
تزكيز العثصر الثقيّل في الزراسب

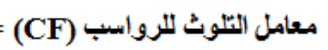

\section{تركيز العنصر الثقّيل في الزراسب القيّاسي}

يمثل جدول ( V) قيم معامل التلوث للعناصر النقيلة في عظيم ا اذ إل سجلت اعلى القيم للعناصر الرصاص والنيكل والنحاس والكروم والحديد

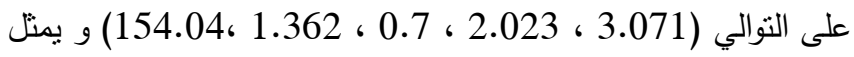

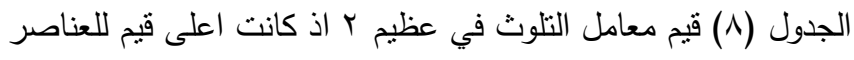

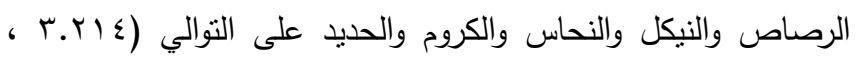

$$
\text { (1) Y .1)، 1.0، . . E) ، 1.79 }
$$

جدول (V) قيم معامل التلوث في عظيم

\begin{tabular}{|c|c|c|c|c|c|}
\hline الستق (سا) & أروصاع CF & CF & لمش CF & حتب CF & الحس CF \\
\hline $0-5$ & 2.785 & 0.490 & 0.25 & 0.714 & 154.04 \\
\hline 5-10 & 2.857 & 0.637 & 0.3 & 0.976 & 149.05 \\
\hline $10-15$ & 2.357 & 0.941 & 0.416 & 1.476 & 138.24 \\
\hline $15-20$ & 2.357 & 0.96 & 0.366 & 1.5 & 132.89 \\
\hline $20-25$ & 2.785 & 0.49 & 0.15 & 0.5 & 135.68 \\
\hline $25-30$ & 2.928 & 0.49 & 0.15 & 0.440 & 11596 \\
\hline $30-35$ & 2.857 & 0.49 & 0.133 & 0.380 & 119.12 \\
\hline $35-40$ & 2.928 & 0.49 & 0.133 & 0.428 & 102.11 \\
\hline $40-45$ & 3.071 & 0.49 & 0.166 & 0.535 & 74.618 \\
\hline $45-50$ & 2.571 & 1.078 & 0.55 & 1.547 & 81.58 \\
\hline $50-55$ & 1.785 & 1.362 & 0.616 & 2.023 & 110.81 \\
\hline $55-60$ & 1.785 & 1.274 & 0.6 & 1916 & 108.38 \\
\hline $60-65$ & 1.785 & 0.598 & 0.416 & 1.047 & 67.602 \\
\hline $65-70$ & 1.785 & 0.49 & 0.383 & 0.916 & 95.204 \\
\hline $70-75$ & 1.785 & 0.49 & 0.416 & 0.916 & 87.637 \\
\hline $75-80$ & 2.785 & 0.49 & 0.2 & 0.630 & 86.163 \\
\hline $80-85$ & 2.642 & 0.49 & 0.166 & 0.523 & 67.690 \\
\hline $85-90$ & 2.785 & 0.49 & 0.166 & 0.511 & 52.468 \\
\hline 90-95 & 1.785 & 0.607 & 0.433 & 0.988 & 59.094 \\
\hline $95-100$ & 1.785 & 0.941 & 0.7 & 1.511 & 48.134 \\
\hline $100-105$ & 1.785 & 0.794 & 0.683 & 1.5 & 46.056 \\
\hline
\end{tabular}


الطينية والغرينية لكونه يمتز على اسطح المعادن الطينية والمواد

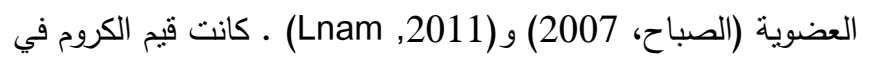

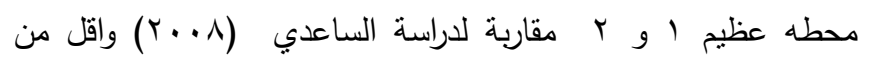
دراسة Alabaychi (1990) الا ان هناك بعض الاعماق في عظيم

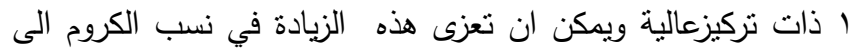

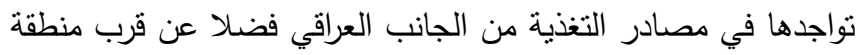
الدراسة الى حقل حلفاية الواقع غرب هور العظيم وقد يعزى زيادة عنصر النيكل والكروم الى زيادة نشاط الصناعات الكيمياوية كمصفى ميسان و مصانع الورق والبلاستك وغيرها من الصناعات المنتشرة في عموم محافظة ميسان فضلا عن نسبة وجوده الطبيعي في التربة كما كانت قيم عنصر النحاس لمنطقة الدراسة اقل من الدراسات السابقة. وقد تعود زيادة عنصر الحديد الى فعل النشاطات الصناعية وخاصة

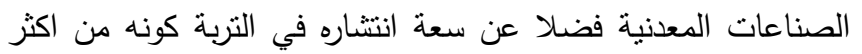
العناصر انتشارا في القترة الارضية (الصرائفي ،9.9. . ب). جدول (9 ) المعايير المعتمدة حالياً للتربة الملوثة بالعناصرالثقيلة

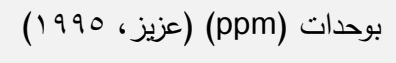

\begin{tabular}{|c|c|c|c|}
\hline Elements & ترب قياسية & الحـ الحرع & ترب ملوثة \\
\hline $\mathbf{P b}$ & 50 & 150 & 600 \\
\hline $\mathrm{Ni}$ & 50 & 100 & 500 \\
\hline $\mathbf{C r}$ & 100 & 250 & 800 \\
\hline $\mathrm{Cu}$ & 20 & 20 & 800 \\
\hline
\end{tabular}

جدول (• ( ) قيم العناصرالثقيلة للدراسات السابقة و منطقة الدراسة

\begin{tabular}{|c|c|c|c|c|c|c|}
\hline ائمسر & $\mathrm{Fe}$ & $\mathrm{Cu}$ & $\mathrm{Pb}$ & $\mathrm{Cr}$ & $\mathrm{Ni}$ & تشفلقة أثراسة \\
\hline Abaychi(1995) & $3606-2721$ & $40-31$ & - & $156-144$ & $212-157$ & هيز انعسر \\
\hline $\begin{array}{l}\text { AL-Saad } \\
\text { etal,2007 }\end{array}$ & 7147.05 & 14.04 & - & - & 44.98 & خهن الزيير \\
\hline الساتثي،2008 & - & 50.85 & 14.7 & 89.69 & 158.3 & هيز البكة \\
\hline \multirow{4}{*}{ AL-Saad, 2009} & 7500 & 16.36 & - & - & 68.3 & هوز ام الثزيد \\
\hline & 6923 & 20.3 & - & - & 52.2 & الم التغاع \\
\hline & 9910 & 32.1 & - & - & 83.3 & البغاية \\
\hline & 7880 & 30.76 & _- & _- & 77.6 & اثيركة \\
\hline $\begin{array}{l}\text { Sultan, } \text {, tall, } \\
2013\end{array}$ & $632.18-980.45$ & $30.12-51.2$ & $1.52-0.62$ & - & - & خهز الززير \\
\hline \multirow{2}{*}{ Present Study } & 5148.14 & 21.14 & 33.3 & 70.85 & 83.95 & هوز أعظيجِا \\
\hline & 5660.47 & 16.05 & 36.1 & 71.05 & 78.82 & شيز أتسئي 2 \\
\hline
\end{tabular}

اعتمد على تركيز العناصر في القتره الارضيه و وعنبرت قيمه مرجعيه وستخدم تصنيف ( Muller, 1979 ) في حساب التجمع الجيوكيميائي
قيم التلوث في منطقة الدراسة. عند مقارنة الدراسة الحاليه مع الدراسات السابقة كما في جدول (•(1) نجد ان عنصر الرصاص فيطة في محطه

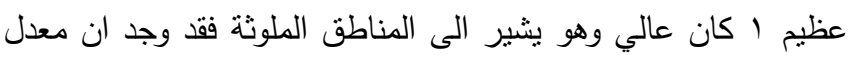
تركيز الرصاص بهور الجكة كان اقل من معدله في منطقه الدراسه

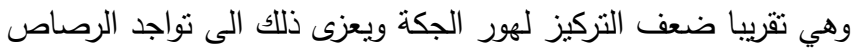

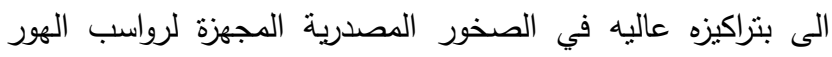

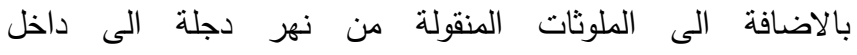

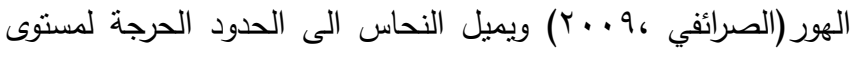

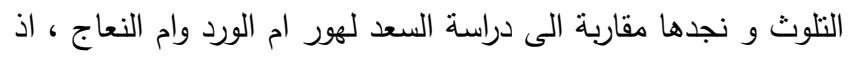

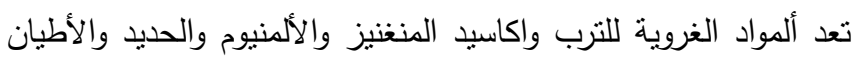
والدبال تمتز عنصرالنحاس بقوة وعلى نحو منزايد عند زيادة الأس

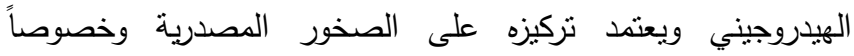

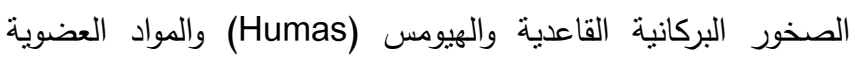
وتركيز الطين (Pope et al.,2011) . يشير عنصر النيكل الى الحد والى

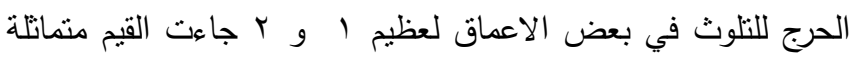

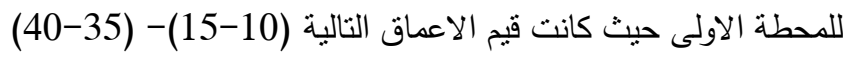

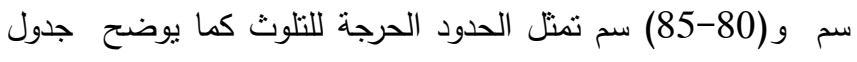

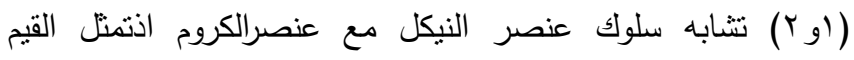

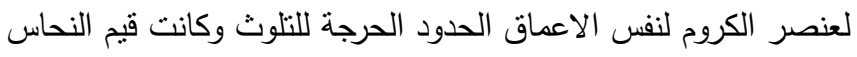
في منطقة عظيم ا تقوق نطاق الحد الحرج للتلوث بينما كانت منطقة عظيم r تقع ضمن نطاق الحد الحرج للترب الملوثة ل ـ وعند مقارنة نتائج قيم العناصر لنقيله في هور العظيم مع دراسة الساعدي لهور

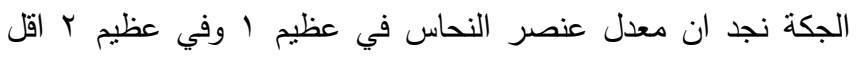

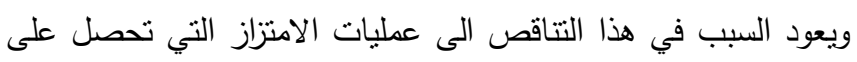
اسطح المعادن الطينية (Bonito, 2005 ) الى عمليات الاكسدة و ولني

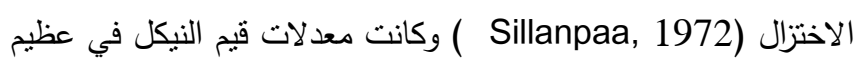

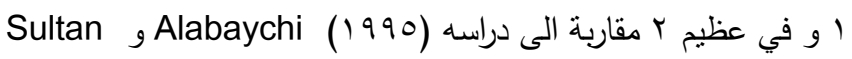

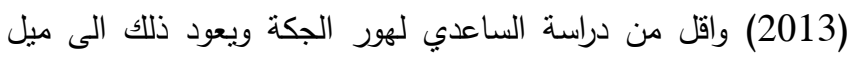

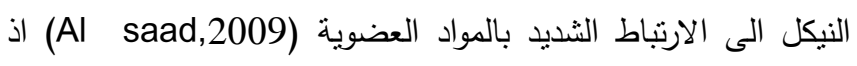
يعتمد تركيزه على وفرة المعادن الطينية والمواد العضوية فهو يدخل بلهن

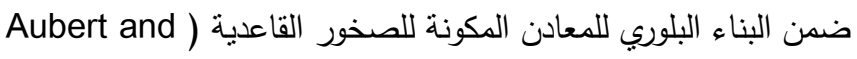
Pinta,1977 ) . ان زيادة نسب النيكل في بعض الاعماق قد يعود

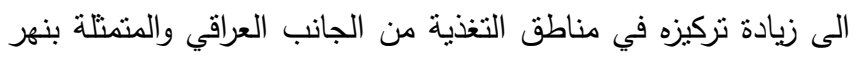

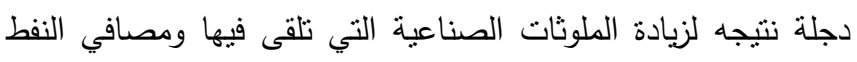
(العزاوي (1996، ) اذ بظهر النيكل علاقة قوية مع حجم الحبيبات 
استخدم تصنيف (Pekey et al.,2004) الجدول (13) في تحديد مقدار نسبة التلوث للرواسب كما كانت نتائج لمقارنة قيم التلوث في جدول (7) و (^) مع تصنيف (Pekey et al.,2004) يظهر تلوث بعض اعماق المنطقة بعنصر الحديد والرصاص وبالدرجة الاقل ياتي النيكل والكروم بينما كان عنصر النحاس لكافة المنطقة غير ملوثة

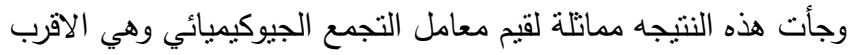
لقيم التراكيز المستحصلة من عمليات القياس . عند استخدام معامل التركيز لرواسب منطقة الدراسة اتخذت العناصر الترتيب التالي

$\mathrm{Fe}>\mathrm{Pb}>\mathrm{Ni}>\mathrm{Cr}>\mathrm{Cu}$ تعزى زيادة نسبة الرصاص الى طبيعة و تركيب الملوثات التي تطرحها

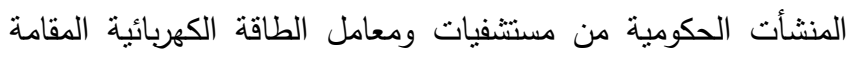

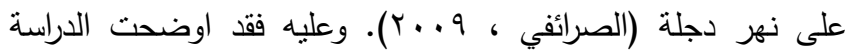

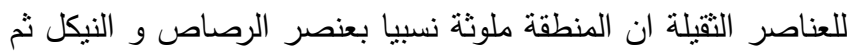
الكروم وغير ملوثة بعنصر النحاس. جدول (r I (Pekey) ) لمعامل التلوث

\begin{tabular}{|c|c|c|}
\hline 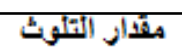 & (CF) قيخ معامل ألتألوث & المجهوعه \\
\hline 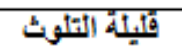 & $\mathrm{CF}<1$ & 1 \\
\hline 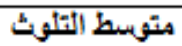 & $1<\mathrm{CF}<3$ & 2 \\
\hline عالئية التهأوث & $3<\mathrm{CF}<6$ & 3 \\
\hline شدياة التلوثث & $\mathrm{CF}>6$ & 4 \\
\hline
\end{tabular}

\section{References}

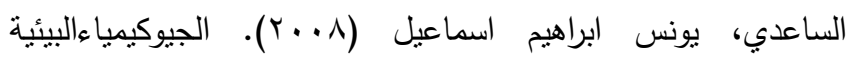

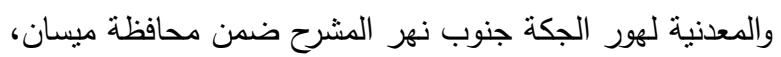

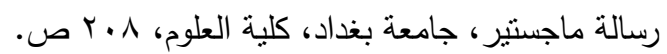

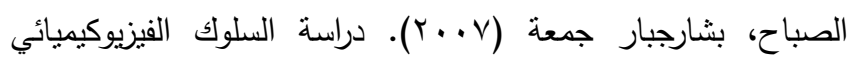
للعناصر المعدنية الملوثة لمياه ورواسب شط العرب. اطروحة

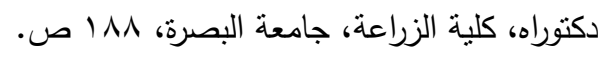

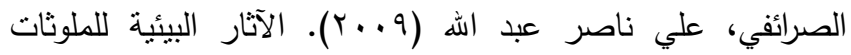
الصناعية في محافظة ميسان. رسالة ماجستير . كلية الاداب -

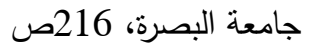

(Audry etal, 2004) عنصر النيكل والكروم والنحاس منتشابهه في السلوك الجيوكيميائي

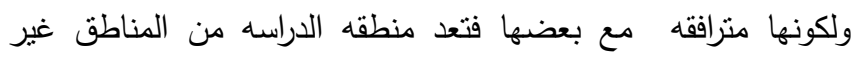
الملوثه الى قليله التلوث مقارنه مع لقيم التجمع الجيوكيميائي في محطة فئه فئه

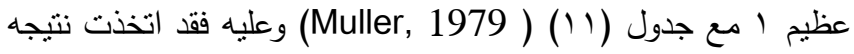
معامل التجمع الجيوكيميائي للعناصر الثقيله الترتيب التالي $\mathrm{Fe}>\mathrm{Pb}>\mathrm{Ni}>\mathrm{Cr}>\mathrm{Cu}$

Muller , جدول (1979) (19)

\begin{tabular}{|c|c|}
\hline دزجة تلوبح الرواسب & Igeo \\
\hline 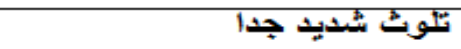 & 5 \\
\hline 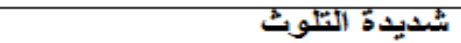 & $5-4$ \\
\hline ملوبتهة بتُّدة & $4-3$ \\
\hline 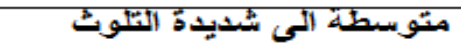 & $3-2$ \\
\hline متوسطة التلّوتُ & $2-1$ \\
\hline 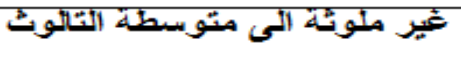 & $\mathbf{1}-\mathbf{0}$ \\
\hline تَّير ملويتة & $<0$ \\
\hline
\end{tabular}

استخدم تصنيف (Sutherland, 2000 ) في تحديد درجة الاغناء للعناصر التقيلة في منطقة الدراسة الجدول (12) فقد اظهرت مقارنة

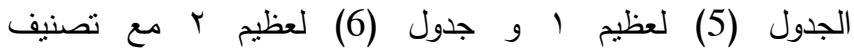
Sutherland

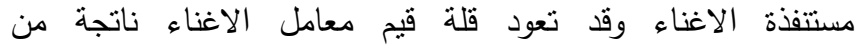
التراكيزالعالية لعنصر الحديد مقارنة مع تراكيز العناصر الثقيلة الاخرى وكان ترتيب معامل اغناء الرواسب بالعناصر النقيلة كالتالي : E.F Fe > E.F Pb > E.F Ni > E.F Cr > E.F Cu غيران نتائج قيم معامل التجمع الجيوكيميائي للعناصر التقيلة اظهرت

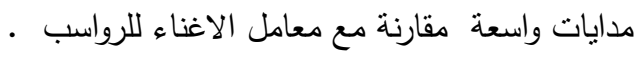
جدول (r I ) تصنيف Sutherland دليل قيم الاغناء بالعناصر الثقيلة

\begin{tabular}{|c|c|}
\hline دكيل معامل الاغناء & لدرجة الاغتاء \\
\hline $\mathbf{E F}<2$ & مستخت: الاغناء \\
\hline $2<\mathbf{E F}<5$ & نسبة مهمة من الاغناء \\
\hline $20<\mathrm{EF}<40$ & نسبة عالية جدا من الاغناء \\
\hline$E F>40$ & منطقةتُشيدة الاغناء \\
\hline
\end{tabular}


Bonito, M.D. (2005). Trace elements in soil pore water: a comparison of sampling methods. Ph.D., University of Nottingham, 263pp.

Guevara, S.R., Massaferro, J., Villarosa, G., Arribere, M. and Rizzo, A. (2002). Heavy metal contamination in sediments of lake nahuel huapi national park, Northern Patagonia, Argentina. Water, Air and Soil pollution, 137: 21-44.

Inam, E., Khantotong, S., Kim, K. and Tumendemberd, B. (2011). Geochemical distribution of trace element Concentrations in the Vicinity of Boroo gold mine, Selenge Province, Mongolia. Environ. Geochem. Health, 33: 57-69.

McDonald, D.G. and Wood, C.M. (1993). Branchial mechanisms of acclimation to metals in fresh water fish (Cliff, R.J. and Jensen, F.B., eds.). Chapman and Hall, London, pp: 299-313.

Muller, G. (1979). Schwemetalle in den Sedimenten ds Rheas-Veranderungen seit. Umschav, 79: 133149.

Pekey, H., Karakas, D., Aybert, S., Tohun, L. and Bakoglu, M. (2004). Ecological risk assessment using trace elements from surface sediment of Izmit Bay (Northeastern Marmara Sea). Turkey. Mar. Pollut. Bull., 48: 946-953.

Pope, N.D. and Langston, W.J. (2011). Sources Distribution and temporal variability of trace metals in the Thames estuary. Hydrobiologia, 672: 49-68.

Sarvides, C., Papadopoulos, A., Haralambous, K.S. and Loizidon, M. (1995). Sediment contaminated with heavy metals: Metal speciation and removal. Water Sci. Tech., 32(9-10): 65-73.

Sillanpää, M. (1972). Trace elements in soil and agriculture. FAO, Soil Bulletin, 17, FAO of UN.

Sultan, A.A., Alhello, A., Aribi, M.A. and Al-Saad, H.T. (2013). Assessment of hydrocarbon and trace metals pollution in waters and sediment of fertilizer company waters in Khor Al-Zubair, Iraq. Mesopot. J. Mar. Sci., (In press).

Sutherland, R.A. (2000). Bed sediment-associated trace metals in an urban stream, Oahu, Hawaii. Environmental Geology, 39(6): 611-637.

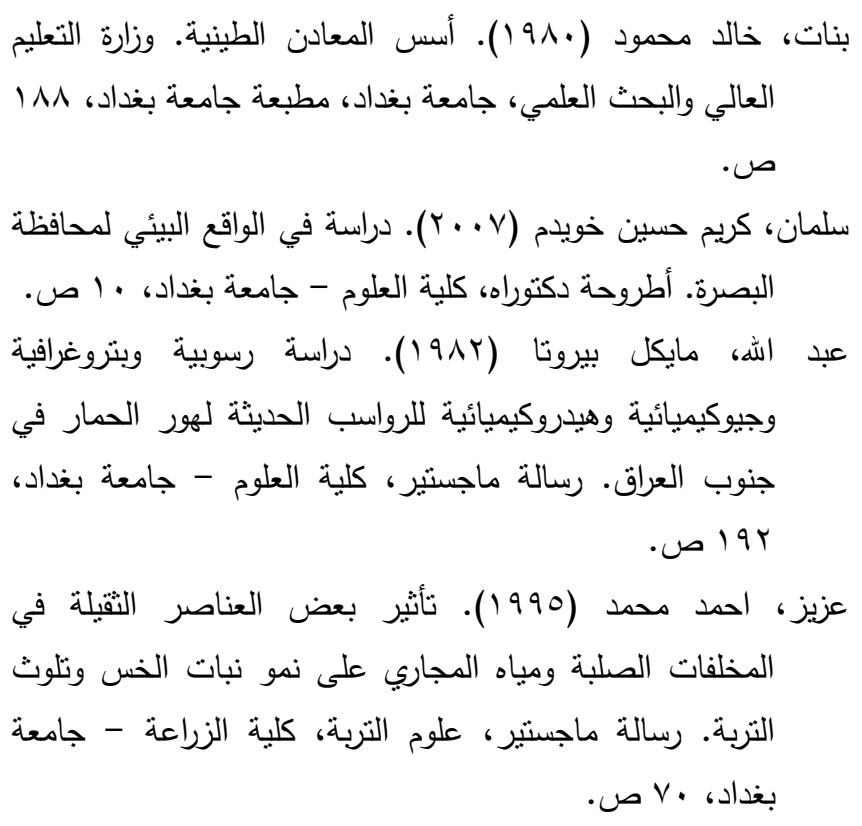

Abaychi, J.K. (1995). Trace elements distribution and sedimentation rate in Al-Hammar lake, Southern Iraq, Marina Mesopotamica, 10(2): 379-392.

Al-Saad, H.T., Abd, I.A., Al-Hello, M.A., and Zuhkair, M.K. (2007). Environmental assessment of trace metals pollution in sediment of Khor AlZubair, Iraq. Marina Mesopotamica, 22(1): 81-92.

Al-Saad, H.T., Al-Taein, S.M., Al-Hello, M.A.R. and DouAbul, A.A.Z. (2009). Hydrocarbons and trace elements in water and Sediments of the marsh Land of Southern Iraq. Mesopotamian Journal of Marine Science, 24(2): 126-139.

Aubert, H. and Pinta, M. (1977). Trace elements in soils. Elsevier scientific publishing company Amsterdam - Oxford - New York, 395pp.

Audry, S., Jorg, S. and Gerard, B. (2004). Fifty-years sedimentary record of heavy metal pollution $(\mathrm{Cd}$, $\mathrm{Zn}, \mathrm{Cu}, \mathrm{Pb}$ ) in the lot river reservoirs (France). Environ. Pollut, p: 498-512.

Awad, N.A.A. and Abdul-Sahib, H.T. (2007). Determination of mercury in the aquatic plants, water and sediments of the southern marshes of Iraq (Al-Amarah and Al-Basrah) and Shatt AlArab river by cold vapour Atomic Absorption Spectromrtry. Marsh Bulletin, 2(2): 137-146.

Botte, S., Freige, R.H. nd Marcovecchio, J.E. (2010). Distribution of several heavy metals in tidal flats sediments within Bahia Blance Estuary (Argentiva). Water Air Soil Pollut., 210: 371-388. 